\title{
Fe II Emission as a Probe of Iron Abundance at Low-z Quasars
}

\author{
Ekaterina Verner \\ $N A S A / G S F C$ and $C U A, U S A$
}

\begin{abstract}
Whether the $\mathrm{Fe} / \alpha$ enrichment is due to $\mathrm{SN}$ Ia explosions or massive objects is a key problem to be addressed in constructing our picture of galactic evolution in the Early Universe. Observations in the rest-frame of $\mathrm{Mg}$ II emission at $2800 \AA$ and the broad emission complex of Fe II spanning 2000-3000 $\AA$ in high-z quasars should allow to determine the time in the Universe when increases in Fe abundance occur. This in turn provides us constraints on the stellar initial mass function and QSO formation and even the cosmological parameters. However a problem remains in developing a reliable model for this complex ion that accurately predicts the emission line spectra seen in luminous AGN at high redshift. The Fe II/Mg II emission ratios using new 830-level Fe II model are investigated with the intent to explain the observed Fe II/Mg II emission scatter.
\end{abstract}

\section{Introduction}

The observed ratio of the ultraviolet Fe II UV emission flux $(2000-3000 \AA$; hereafter Fe II UV ) to that of the Mg II resonance doublet at $2800 \AA$ in broadline regions (BLRs) of quasars has a strong potential for being a fundamental cosmological metallicity indicator. In models of galactic chemical evolution, the ratio of Fe to $\alpha$-element $(\mathrm{O}, \mathrm{Ne}, \mathrm{Mg})$ abundances constrains the ages of starforming systems. The $\mathrm{Fe} / \alpha$ age constraint follows from a different enrichment timescale, namely: $\alpha$-elements are produced in supernova explosions of shortlived massive stars (primarily SN Type II), while Fe has a large contribution from the longer-lived intermediate-mass binaries that produce Type Ia supernovae. Predictions indicate that the $\mathrm{Fe} / \alpha$ abundance ratio should increase by more than an order of magnitude for stellar systems older than 1 Gyr (Hamann \& Ferland 1993; Yoshii et al. 1996). Other theoretical explanations for producing Fe are also possible (Heger \& Woosley 2002).

\section{Fe II UV/Mg II Emission Ratios}

Since many quasars simultaneously exhibit prominent Fe II UV and Mg II resonance doublet emission in their spectra, all previous observational efforts focused on Fe II UV/Mg II emission measurements and their dependence on redshift. Yet no evolution effect of Fe II UV/Mg II up to $\mathrm{z} \sim 6.4$ has been found (Kawara et al. 1996; Iwamuro et al. 2002; Dietrich et al. 2002; Freudling et al. 2003; 

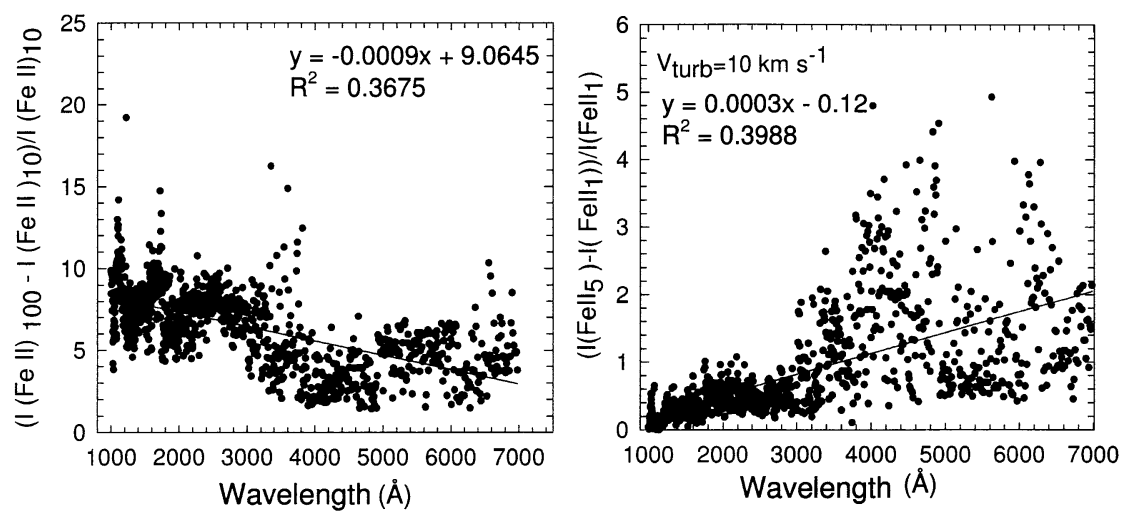

Figure 1. The general sensitivity of the Fe II continuum to changes in turbulence and abundance (Verner et al. 2003).

Barth et al. 2003). Meanwhile due to the complex environments, modeling is needed to obtain accurate abundances from the spectra. Recent studies based on Fe II modeling predict that Fe II (Optical) band (4000-6000 $\AA$; thereafter Fe II (Optical) is more sensitive to abundance than the Fe II UV band (Figure 1; Verner et al. 2003). The modeling reveals that interpretations of high values of Fe II UV/Mg II are sensitive not only to Fe and $\mathrm{Mg}$ abundance, but also to other factors such as microturbulence, density, and properties of the radiation field. We find that the Fe II UV/Mg II ratio combined with Fe II UV/Fe II (Optical) emission ratio can be used as a reliable nucleosynthesis diagnostic for the $\mathrm{Fe} / \mathrm{Mg}$ abundance ratios for the physical conditions relevant to the broad-line regions of quasars.

Acknowledgments. EV acknowledges financial support from the NSF via grant NSF 0206150 and travel support to IAUXXV provided by the AAS.

\section{References}

Barth, A. J., Martini, P., Nelson, C., \& Ho, L., 2003, ApJ, 594, L95.

Dietrich, M., et al., 2002, ApJ, 564, 581.

Freudling, W., Corbin, M., \& Korista, K. 2003, ApJ, 587, L67

Hamann, F., \& Ferland, G. 1993, ApJ, 418, 11.

Heger, A., \& Woosley, S. E. 2002, ApJ, 567, 532

Iwamuro, F., et al., 2002, ApJ, 565, 63

Kawara, K., Murayama, T., Taniguchi, Y., \& Arimoto, N. 1996, ApJ, 470, L85.

Yoshii, Y., Tsujimoto, T., \& Kawara, K. 1998, ApJ, 507, L113.

Verner, E., et al., 2003, ApJ, 592, L59. 